\title{
On Fractional Order Dengue Epidemic Model
}

\author{
Hamed Al-Sulami, ${ }^{1}$ Moustafa El-Shahed,, ${ }^{1,2}$ Juan J. Nieto, ${ }^{1,3}$ and Wafa Shammakh ${ }^{1}$ \\ ${ }^{1}$ Department of Mathematics, Faculty of Science, King Abdulaziz University, P.O. Box 80203, Jeddah 21589, Saudi Arabia \\ ${ }^{2}$ Department of Mathematics, Faculty of Art and Sciences, Qassim University, P.O. Box 3771, Unaizah-Qassim 51911, Saudi Arabia \\ ${ }^{3}$ Departamento de Analisis Mateatico, Facultad de Matematicas, Universidad de Santiago de Compostela, \\ 15782 Santiago de Compostela, Spain
}

Correspondence should be addressed to Hamed Al-Sulami; hamed9@hotmail.com

Received 17 April 2014; Accepted 23 June 2014; Published 18 August 2014

Academic Editor: J. A. Tenreiro Machado

Copyright (C) 2014 Hamed Al-Sulami et al. This is an open access article distributed under the Creative Commons Attribution License, which permits unrestricted use, distribution, and reproduction in any medium, provided the original work is properly cited.

This paper deals with the fractional order dengue epidemic model. The stability of disease-free and positive fixed points is studied. Adams-Bashforth-Moulton algorithm has been used to solve and simulate the system of differential equations.

\section{Introduction}

Dengue is a major public health problem in tropical and subtropical countries. It is a vector-borne disease transmitted by Aedes aegypti and Aedes albopictus mosquitoes. Four different serotypes can cause dengue fever. A human infected by one serotype, when recovers, gains total immunity to that serotype and only partial and transient immunity with respect to the other three.

Dengue can vary from mild to severe. The more severe forms of dengue include shock syndrome and dengue hemorrhagic fever (DHF). Patients who develop these more serious forms of dengue fever usually need to be hospitalized. The full life cycle of dengue fever virus involves the role of the mosquito as a transmitter (or vector) and humans as the main victim and source of infection. Preventing or reducing dengue virus transmission depends entirely on the control of mosquito vectors or interruption of human vector contact $[1,2]$.

In this paper we study the fractional order dengue epidemic model. The stability of equilibrium points is studied. Numerical solutions of this model are given. We like to argue that fractional order equations are more suitable than integer order ones in modeling biological, economic, and social systems (generally complex adaptive systems) where memory effects are important. Adams-Bashforth-Moulton algorithm has been used to solve and simulate the system of differential equations.

\section{Model Derivation}

Esteva and Vargas [3] developed a dengue fever transmission model by assuming that, once a person recovers from the disease, he or she will not be reinfected by the disease. The model also assumes that the host population $N_{h}$ is constant, that is, the death rate and the birth rate equal $\mu_{H}$. The hostvector model for the dengue transmission of Esteva and Vargas [3] is as follows:

$$
\begin{gathered}
\frac{d S_{h}}{d t}=A-\frac{\beta_{h} b}{N_{h}} S_{h} I_{v}-\mu_{h} S_{h}, \\
\frac{d I_{h}}{d t}=\frac{\beta_{h} b}{N_{h}} S_{h} I_{v}-\left(\mu_{h}+\gamma\right) I_{h}, \\
\frac{d R_{h}}{d t}=\gamma I_{h}-\mu_{h} R_{h}, \\
\frac{d S_{v}}{d t}=B-\frac{\beta_{v} b}{N_{h}} S_{v} I_{h}-\mu_{v} S_{v}, \\
\frac{d I_{v}}{d t}=\frac{\beta_{v} b}{N_{h}} S_{v} I_{h}-\mu_{v} I_{v},
\end{gathered}
$$

where

$A$ is the recruitment rate of the host population, $B$ is the recruitment rate of the vector population, 
$S_{h}$ is the number of susceptible in the host population,

$I_{h}$ is the number of infective in the host population,

$R_{h}$ is the number of immunes in the host population,

$N_{v}$ is the vector population,

$S_{v}$ is the number of susceptible in the vector population,

$I_{v}$ is the number of infective in the vector population,

$\mu_{v}$ is the death rate in the vector population,

$\beta_{h}$ is the transmission probability from vector to host,

$\beta_{v}$ is the transmission probability from host to vector,

$\gamma$ is the recovery rate in the host population,

$b$ is the biting rate of the vector.

The notion of fractional calculus was anticipated by Leibniz, one of the founders of standard calculus, in a letter written in 1695. Recently great considerations have been made to the models of FDEs in different aria of researches. The most essential property of these models is their nonlocal property which does not exist in the integer order differential operators. We mean by this property that the next state of a model depends not only upon its current state but also upon all of its historical states. There are many definitions of fractional derivatives $[4,5]$. Perhaps the best-known is the Riemann-Liouvile definition. The Riemann-Liouville derivative of order $\alpha$ is defined as

$$
\begin{array}{r}
\mathrm{RL}_{0+} D_{0}^{\alpha} f(t)=\frac{1}{\Gamma(n-\alpha)}\left(\frac{d}{d t}\right)^{n} \int_{0}^{t} \frac{f(s)}{(t-s)^{\alpha-n+1}} d s, \\
n=[\alpha]+1,
\end{array}
$$

where $\Gamma()$ is the gamma function and $n$ is an integer. An alternative definition was introduced by Caputo as follows, which is a sort of regularization of the Riemann-Liouville derivative:

$$
D_{t}^{\alpha} f(t)=\frac{1}{\Gamma(n-\alpha)} \int_{0}^{t} \frac{f^{(n)}(s)}{(t-s)^{\alpha-n+1}} d s .
$$

Pooseh et al. [6] introduced the notion of fractional derivative in the sense of Riemann-Liouville to reformulate the dynamics of the classical model (1) in terms of fractional derivatives. They applied a recent approximate technique to obtain numerical solutions to the fractional model. The system in this paper will be in the sense of Caputo fractional derivative by the following set of fractional order differential equations:

$$
\begin{gathered}
D_{t}^{\alpha} S_{h}=A-\frac{\beta_{h} b}{N_{h}} S_{h} I_{v}-\mu_{h} S_{h}, \\
D_{t}^{\alpha} I_{h}=\frac{\beta_{h} b}{N_{h}} S_{h} I_{v}-\left(\mu_{h}+\gamma\right) I_{h}, \\
D_{t}^{\alpha} R_{h}=\gamma I_{h}-\mu_{h} R_{h}, \\
D_{t}^{\alpha} S_{v}=B-\frac{\beta_{v} b}{N_{h}} S_{v} I_{h}-\mu_{v} S_{v}, \\
D_{t}^{\alpha} I_{v}=\frac{\beta_{v} b}{N_{h}} S_{v} I_{h}-\mu_{v} I_{v} .
\end{gathered}
$$

Because model (4) monitors the dynamics of human populations, all the parameters are assumed to be nonnegative. Furthermore, it can be shown that all state variables of the model are nonnegative for all time $t \geq 0$ (see, for instance, [7-9]).

Lemma 1. The closed set $\Omega=\left\{\left(S_{h}, I_{h}, R_{h}, S_{v}, I_{v}\right) \in R_{+}^{5}: S_{h}+\right.$ $\left.I_{h}+R_{h}=A / \mu_{h}, S_{v}+I_{v}=B / \mu_{v}\right\}$ is positively invariant with respect to model (4).

Proof. The fractional derivative of the total population, obtained by adding all the equations of model (4), is given by

$$
D_{t}^{\alpha} N_{h}(t)=A-\mu_{h} N_{h}(t)
$$

The solution to (5) is given by $N_{h}(t)=$ $N_{h}(0) E_{\alpha, 1}\left(-\mu_{h} t^{\alpha}\right)+A t^{\alpha} E_{\alpha, \alpha+1}\left(-\mu_{h} t^{\alpha}\right)$, where $E_{\alpha, \beta}$ is the Mittag-Leffler function. Considering the fact that the Mittag-Leffler function has an asymptotic behavior $[4,10]$,

$$
\begin{array}{r}
E_{\alpha, \beta}(z) \sim-\sum_{k=1}^{\omega} \frac{z^{-k}}{\Gamma(\beta-\alpha k)}+O\left(|z|^{-1-\omega}\right), \\
\left(|z| \longrightarrow \infty, \frac{\alpha \pi}{2}<|\arg (z)| \leq \pi\right) .
\end{array}
$$

One can observe that $N_{h}(t) \rightarrow A / \mu_{h}$ as $t \rightarrow \infty$. The proof of vector population case is completely similar to that of host population and is therefore omitted. One can observe that $N_{v}(t) \rightarrow B / \mu_{v}$. Therefore, all solutions of the model with initial conditions in $\Omega$ remain in $\Omega$ for all $t>0$. Thus, region $\Omega$ is positively invariant with respect to model (4).

In the following, we will study the dynamics of system (4).

\section{Equilibrium Points and Stability}

To evaluate the equilibrium points let

$$
\begin{gathered}
D_{t}^{\alpha} S_{h}=0, \quad D_{t}^{\alpha} I_{h}=0, \quad D_{t}^{\alpha} R_{h}=0, \\
D_{t}^{\alpha} S_{v}=0, \quad D_{t}^{\alpha} I_{v}=0
\end{gathered}
$$


Then $E_{0}=\left(A / \mu_{h}, 0,0,\left(B / \mu_{\nu}\right) 0\right)$. By (4), a positive equilibrium $E_{1}=\left(S_{h}^{1}, I_{h}^{1}, R_{h}^{1}, S_{v}^{1}, I_{v}^{1}\right)$ satisfies

$$
\begin{gathered}
S_{h}^{1}=\frac{N_{h} \mu_{v}\left(A b \beta_{v}+N_{h}\left(\gamma+\mu_{h}\right) \mu_{v}\right)}{b \beta_{v}\left(b B \beta_{h}+N_{h} \mu_{h} \mu_{v}\right)}, \\
I_{h}^{1}=\frac{A b^{2} B \beta_{h} \beta_{v}-N_{h}^{2} \mu_{h}\left(\gamma+\mu_{h}\right) \mu_{v}^{2}}{b \beta_{v}\left(\gamma+\mu_{h}\right)\left(b B \beta_{h}+N_{h} \mu_{h} \mu_{v}\right)}, \\
R_{h}^{1}=\frac{A b^{2} B \gamma \beta_{h} \beta_{v}-\gamma N_{h}^{2} \mu_{h}\left(\gamma+\mu_{h}\right) \mu_{v}^{2}}{b \beta_{v} \mu_{h}\left(\gamma+\mu_{h}\right)\left(b B \beta_{h}+N_{h} \mu_{h} \mu_{v}\right)}, \\
S_{v}^{1}=\frac{N_{h}\left(\gamma+\mu_{h}\right)\left(b B \beta_{h}+N_{h} \mu_{h} \mu_{v}\right)}{b \beta_{h}\left(A b \beta_{v}+N_{h}\left(\gamma+\mu_{h}\right) \mu_{v}\right)}, \\
I_{v}^{1}=\frac{A b^{2} B \beta_{h} \beta_{v}-N_{h}^{2} \mu_{h}\left(\gamma+\mu_{h}\right) \mu_{v}^{2}}{b \beta_{h} \mu_{v}\left(A b \beta_{v}+N_{h}\left(\gamma+\mu_{h}\right) \mu_{v}\right)} .
\end{gathered}
$$

The Jacobian matrix $J\left(E_{0}\right)$ for the system given in (4) evaluated at the disease-free equilibrium is as follows:

$$
J\left(E_{0}\right)=\left(\begin{array}{ccccc}
-\mu_{h} & 0 & 0 & 0 & -\frac{A b \beta_{h}}{N_{h} \mu_{h}} \\
0 & -\gamma-\mu_{h} & 0 & 0 & \frac{A b \beta_{h}}{N_{h} \mu_{h}} \\
0 & \gamma & -\mu_{h} & 0 & 0 \\
0 & -\frac{b B \beta_{v}}{N_{h} \mu_{v}} & 0 & -\mu_{v} & 0 \\
0 & \frac{b B \beta_{v}}{N_{h} \mu_{v}} & 0 & 0 & -\mu_{v}
\end{array}\right) .
$$

Theorem 2. The disease-free equilibrium $E_{0}$ is locally asymptotically stable if $R_{0}<1$ and is unstable if $R_{0}>1$.

Proof. The disease-free equilibrium is locally asymptotically stable if all the eigenvalues, $\lambda_{i}, i=1,2,3,4,5$ of the Jacobian matrix $J\left(E_{0}\right)$ satisfy the following condition [11-14]:

$$
\left|\arg \left(\lambda_{i}\right)\right|>\frac{\alpha \pi}{2}
$$

The eigenvalues of the Jacobian matrix $J\left(E_{0}\right)$ are $\lambda_{1}=$ $-\mu_{h}, \lambda_{2}=-\mu_{h}$, and $\lambda_{3}=-\mu_{v}$; the other two roots are determined by the quadratic equation

$$
\lambda^{2}+\lambda\left(\gamma+\mu_{h}+\mu_{v}\right)+\mu_{v}\left(\gamma+\mu_{h}\right)\left(1-R_{0}\right)=0,
$$

where $R_{0}=\left(A B b^{2} \beta_{h} \beta_{v}\right) /\left(\mu_{h} \mu_{v}^{2} N_{h}^{2}\left(\gamma+\mu_{h}\right)\right)$. Hence $E_{0}$ is locally asymptotically stable if $R_{0}<1$ and is unstable if $R_{0}>1$.

The quantity $R_{0}^{*}=\sqrt{R_{0}}$ is called the basic reproductive number of the disease, since it represents the average number of secondary cases that one case can produce if introduced into a susceptible population.
We now discuss the asymptotic stability of the endemic (positive) equilibrium of the system given by (4). The Jacobian matrix $J\left(E_{1}\right)$ evaluated at the endemic equilibrium is given as

$$
J\left(E_{1}\right)\left(\begin{array}{ccccc}
-\frac{b \beta_{h} I_{v}^{1}}{N_{h}}-\mu_{h} & 0 & 0 & 0 & -\frac{b S_{h}^{1} \beta_{h}}{N_{h}} \\
\frac{b \beta_{h} I_{v}^{1}}{N_{h}} & -\gamma-\mu_{h} & 0 & 0 & \frac{b S_{h}^{1} \beta_{h}}{N_{h}} \\
0 & \gamma & -\mu_{h} & 0 & 0 \\
0 & -\frac{b S_{h}^{1} \beta_{v}}{N_{h}} & 0 & -\frac{b \beta_{v} I_{h}^{1}}{N_{h}}-\mu_{v} & 0 \\
0 & \frac{b S_{v}^{1} \beta_{v}}{N_{h}} & 0 & \frac{b \beta_{v} I_{h}^{1}}{N_{h}} & -\mu_{v}
\end{array}\right) .
$$

The characteristic equation of $J\left(E_{1}\right)$ is

$$
\left(\lambda+\mu_{h}\right)\left(\lambda+\mu_{v}\right)\left(\lambda^{3}+a_{1} \lambda^{2}+a_{2} \lambda+a_{3}\right)=0,
$$

where

$$
\begin{aligned}
a_{1}= & \gamma+\mu_{v}+\frac{M_{2}\left(K \theta+M_{1} \mu_{v}\right)}{K \theta M_{1} N_{h} \mu_{v}}, \\
a_{2}= & \left(M_{2}^{2}+K \theta M_{1} N_{h}^{2} \mu_{h} \mu_{v}\left(\theta+\mu_{v}\right)\right. \\
& \left.+M_{2} N_{h}\left(K \theta^{2}+\left(K \theta+M_{1}\left(\theta+\mu_{h}\right)\right) \mu_{v}\right)\right) \\
& \times\left(K \theta M_{1} N_{h}^{2} \mu_{v}\right)^{-1}, \\
a_{3}= & \frac{M_{2}\left(M_{2}+N_{h}\left(K \theta+M_{1} \mu_{h}\right) \mu_{v}\right)}{K M_{1} N_{h}^{2} \mu_{v}}, \\
M_{1}= & A b^{2} B \beta_{h} \beta_{v}-N_{h}^{2} \mu_{h}\left(\gamma+\mu_{h}\right) \mu_{v}^{2}, \\
M_{2}= & A b \beta_{v}+N_{h}\left(\gamma+\mu_{h}\right) \mu_{v}, \\
K= & b B \beta_{h}+N_{h} \mu_{h} \mu_{v}, \\
\theta= & \gamma+\mu_{h} .
\end{aligned}
$$

If $p(x)=x^{3}+a_{1} x^{2}+a_{2} x+a_{3}$. Let $D(p)$ denote the discriminant of a polynomial $p(x)$; then

$$
\begin{aligned}
D(p) & =-\left|\begin{array}{ccccc}
1 & a_{1} & a_{2} & a_{3} & 0 \\
0 & 1 & a_{1} & a_{2} & a_{3} \\
3 & 2 a_{1} & a_{2} & 0 & 0 \\
0 & 3 & a_{1} & a_{2} & 0 \\
0 & 0 & 3 & 2 a_{1} & a_{2}
\end{array}\right| \\
& =18 a_{1} a_{2} a_{3}+\left(a_{1} a_{2}\right)^{2}-4 a_{3} a_{1}^{3}-4 a_{2}^{3}-27 a_{3}^{2} .
\end{aligned}
$$

Following [14-18], we have Proposition 3.

Proposition 3. One assumes that $E_{1}$ exists in $R_{+}^{3}$.

(i) If the discriminant of $p(x), D(p)$, is positive and RouthHurwitz are satisfied, that is, $D(p)>0, a_{1}>0, a_{3}>0$, and $a_{1} a_{2}>a_{3}$, then $E_{1}$ is locally asymptotically stable. 
(ii) If $D(p)<0, a_{1}>0, a_{2}>0, a_{1} a_{2}=a_{3}$, and $\alpha \in[0,1)$, then $E_{1}$ is locally asymptotically stable.

(iii) If $D(p)<0, a_{1}<0, a_{2}<0$, and $\alpha>2 / 3$, then $E_{1}$ is unstable.

(iv) The necessary condition for the equilibrium point $E_{1}$, to be locally asymptotically stable, is $a_{3}>0$.

\section{Numerical Methods and Simulations}

Since most of the fractional order differential equations do not have exact analytic solutions, so approximation and numerical techniques must be used. Several analytical and numerical methods have been proposed to solve the fractional order differential equations. For numerical solutions of the system (4) one can use the generalized Adams-BashforthMoulton method. To give the approximate solution by means of this algorithm, consider the following nonlinear fractional differential equation [19]:

$$
\begin{array}{r}
D_{t}^{\alpha} y(t)=f(t, y(t)), \quad 0 \leq t \leq T, \\
y^{(k)}(0)=y_{0}^{k}, \quad k=0,1,2, \ldots, m-1, \quad \text { where } m=[\alpha] .
\end{array}
$$

This equation is equivalent to Volterra integral equation:

$$
y(t)=\sum_{k=0}^{m-1} y_{0}^{(k)} \frac{t^{k}}{k !}+\frac{1}{\Gamma(\alpha)} \int_{0}^{t}(t-s)^{\alpha-1} f(s, y(s)) d s
$$

Diethelm et al. used the predictor-correctors scheme [15, $16,20]$ based on the Adams-Bashforth-Moulton algorithm to integrate (17). By applying this scheme to the fractional order dengue epidemic model and setting $h=T / N, t_{n}=n h$, and $n=0,1,2, \ldots, N \in Z^{+}$, (17) can be discretized as follows [19]:

$$
\begin{aligned}
S_{n+1}= & S_{0}+\frac{h^{\alpha}}{\Gamma(\alpha+2)}\left(A-\frac{\beta_{h} b_{h}}{N_{h}} S_{n+1}^{p} Y_{n+1}^{p}-\mu_{h} S_{n+1}^{p}\right) \\
& +\frac{h^{\alpha}}{\Gamma(\alpha+2)} \sum_{j=0}^{n} a_{j, n+1}\left(A-\frac{\beta_{h} b_{h}}{N_{h}} S_{j} Y_{j}-\mu_{h} S_{j}\right), \\
I_{n+1}= & I_{0}+\frac{h^{\alpha}}{\Gamma(\alpha+2)}\left(\frac{\beta_{h} b_{h}}{N_{h}} S_{n+1}^{p} Y_{n+1}^{p}-\left(\mu_{h}+\gamma\right) I_{n+1}^{p}\right) \\
& +\frac{h^{\alpha}}{\Gamma(\alpha+2)} \sum_{j=0}^{n} a_{j, n+1}\left(\frac{\beta_{h} b_{h}}{N_{h}} S_{j} Y_{j}-\left(\mu_{h}+\gamma\right) I_{j}\right), \\
R_{n+1}= & R_{0}+\frac{h^{\alpha}}{\Gamma(\alpha+2)}\left(\gamma I_{n+1}^{p}-\mu_{h} R_{n+1}^{p}\right) \\
& +\frac{h^{\alpha}}{\Gamma(\alpha+2)} \sum_{j=0}^{n} a_{j, n+1}\left(\gamma I_{j}-\mu_{h} R_{j}\right),
\end{aligned}
$$

$$
\begin{aligned}
X_{n+1}= & X_{0}+\frac{h^{\alpha}}{\Gamma(\alpha+2)}\left(B-\frac{\beta_{v} b_{h}}{N_{h}} X_{n+1}^{p} I_{n+1}^{p}-\mu_{h} X_{n+1}^{p}\right) \\
& +\frac{h^{\alpha}}{\Gamma(\alpha+2)} \sum_{j=0}^{n} a_{j, n+1}\left(B-\frac{\beta_{v} b_{h}}{N_{h}} X_{j} I_{j}-\mu_{v} X_{j}\right), \\
Y_{n+1}= & Y_{0}+\frac{h^{\alpha}}{\Gamma(\alpha+2)}\left(\frac{\beta_{v} b_{h}}{N_{h}} X_{n+1}^{p} I_{n+1}^{p}-\mu_{h} Y_{n+1}^{p}\right) \\
& +\frac{h^{\alpha}}{\Gamma(\alpha+2)} \sum_{j=0}^{n} a_{j, n+1}\left(\frac{\beta_{v} b_{h}}{N_{h}} X_{j} I_{j}-\mu_{v} Y_{j}\right),
\end{aligned}
$$

where

$$
\begin{aligned}
& S_{n+1}^{p}=S_{0}+\frac{1}{\Gamma(\alpha)} \sum_{j=0}^{n} b_{j, n+1}\left(A-\frac{\beta_{h} b_{h}}{N_{h}} S_{j} Y_{j}-\mu_{h} S_{j}\right), \\
& I_{n+1}^{p}=I_{0}+\frac{1}{\Gamma(\alpha)} \sum_{j=0}^{n} b_{j, n+1}\left(\frac{\beta_{h} b_{h}}{N_{h}} S_{j} Y_{j}-\left(\mu_{h}+\gamma\right) I_{j}\right), \\
& R_{n+1}^{p}=R_{0}+\frac{1}{\Gamma(\alpha)} \sum_{j=0}^{n} b_{j, n+1}\left(\gamma I_{j}-\mu_{h} R_{j}\right), \\
& X_{n+1}^{p}=X_{0}+\frac{1}{\Gamma(\alpha)} \sum_{j=0}^{n} b_{j, n+1}\left(B-\frac{\beta_{v} b_{h}}{N_{h}} X_{j} I_{j}-\mu_{v} X_{j}\right) \text {, } \\
& Y_{n+1}^{p}=Y_{0}+\frac{1}{\Gamma(\alpha)} \sum_{j=0}^{n} b_{j, n+1}\left(\frac{\beta_{v} b_{h}}{N_{h}} X_{j} I_{j}-\mu_{v} Y_{j}\right), \\
& a_{j, n+1}=\left\{\begin{array}{cl}
n^{\alpha+1}-(n-\alpha)(n+1), & j=0, \\
(n-j+2)^{\alpha+1}+(n-j)^{\alpha+1} & 1 \leq j \leq n, \\
-2(n-j+1)^{\alpha+1}, & j=n+1,
\end{array}\right. \\
& b_{j, n+1}=\frac{h^{\alpha}}{\alpha}\left((n-j+1)^{\alpha}-(n-j)^{\alpha}\right), \quad 0 \leq j \leq n .
\end{aligned}
$$

\section{Discussion}

In this paper, we have considered a fractional calculus model for dengue disease. Following [21], Figure 1 shows that $S_{h}$ drops significantly in a relatively small period of time. Both $I_{h}$ and $I_{v}$ increase significantly during the period of 30 days and then eventually oscillate around the endemic state $(0.09529$, 0.0.00029, and 0.00058). This seems unrealistic in the nature. With constant population of mosquitoes, this fluctuation (in a short period of time) cannot be shown to happen in the nature [21]. As mentioned by [6], Figures 2 and 3 show that even a simple fractional model may give surprisingly good results. However, the transformation of a classical model into a fractional one makes it very sensitive to the order of differentiation $\alpha$ : a small change in $\alpha$ may result in a big change in the final result. From the numerical results in Figures 2 and 3, it is clear that the approximate solutions depend continuously on the fractional derivative $\alpha$. 

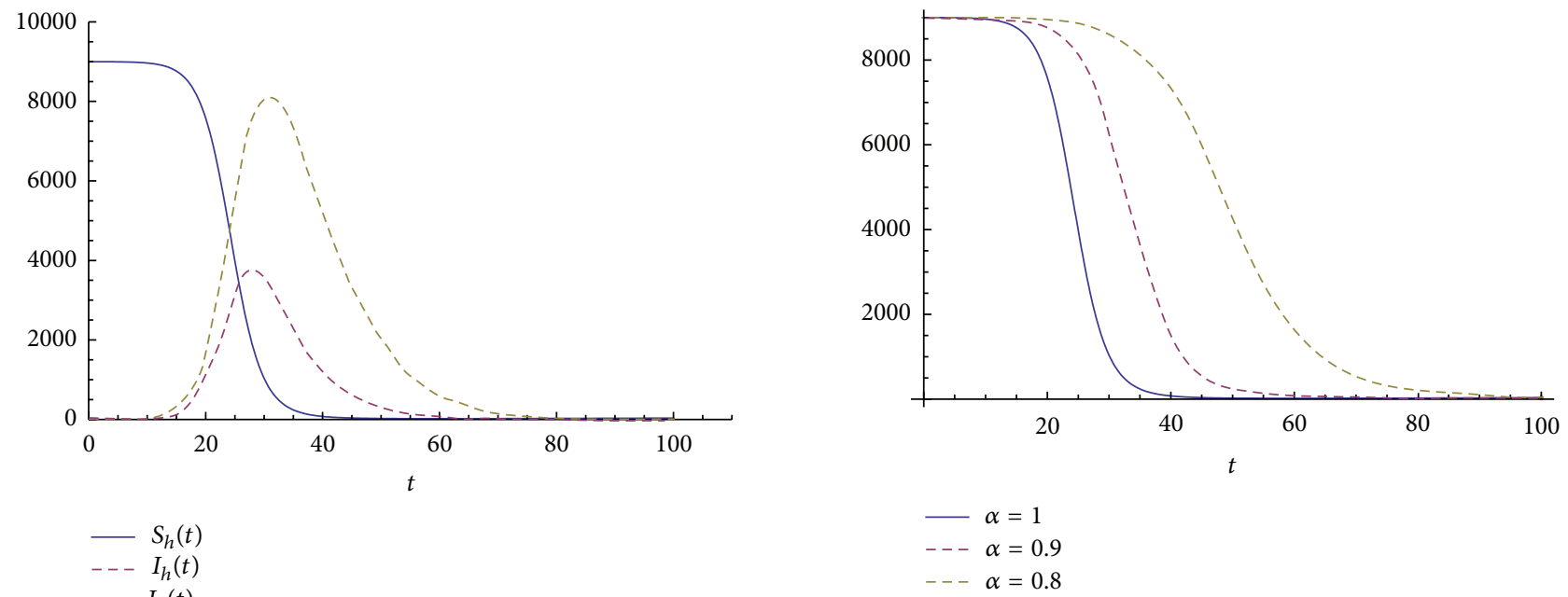

FIGURE 1: $S_{h}(t), I_{h}(t)$, and $I_{v}(t)$ for $\alpha=1$ and $\mu_{h}=0.0000457 ; \mu_{v}=$ $0.25 ; b=0.5 ; \beta_{h}=0.75 ; \beta_{v}=1 ; \gamma=0.1428 ; N_{h}=10000 ; B=5000$.

FIgURE 4: $S_{h}(t)$ for $\alpha=1,0.9,0.8$ and $\mu_{h}=0.0000457 ; \mu_{v}=0.25$; $b=0.5 ; \beta_{h}=0.75 ; \beta_{v}=1 ; \gamma=0.1428 ; N_{h}=10000 ; B=5000$.

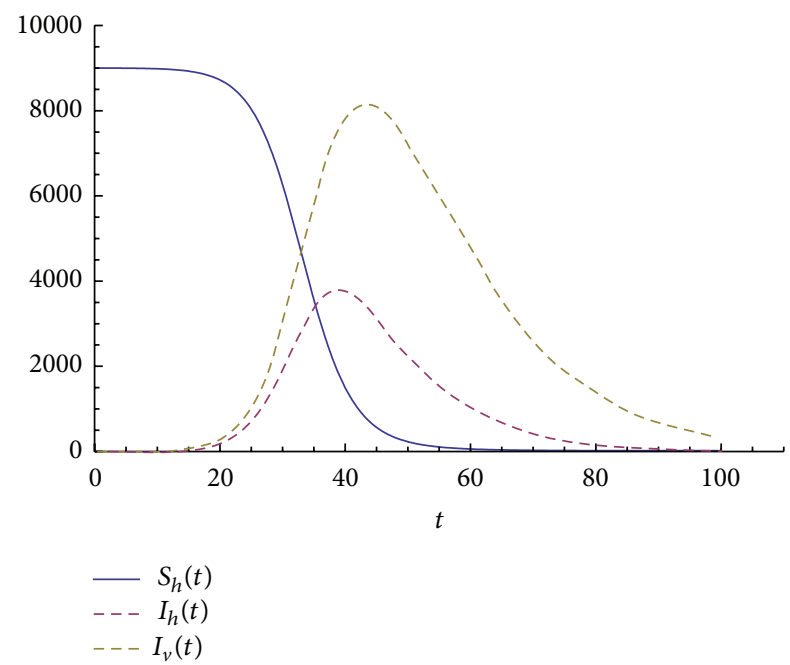

Figure 2: $S_{h}(t), I_{h}(t)$, and $I_{v}(t)$ for $\alpha=0.9$ and $\mu_{h}=0.0000457$; $\mu_{v}=0.25 ; b=0.5 ; \beta_{h}=0.75 ; \beta_{v}=1 ; \gamma=0.1428 ; N_{h}=10000$; $B=5000$.

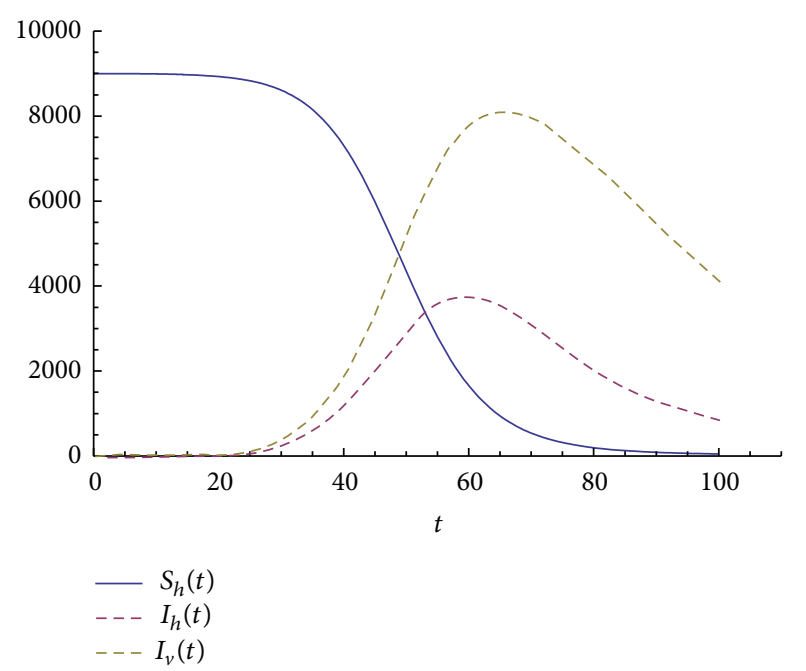

FIGURE 3: $S_{h}(t), I_{h}(t)$, and $I_{v}(t)$ for $\alpha=0.8$ and $\mu_{h}=0.0000457$; $\mu_{v}=0.25 ; b=0.5 ; \beta_{h}=0.75 ; \beta_{v}=1 ; \gamma=0.1428 ; N_{h}=10000$; $B=5000$.

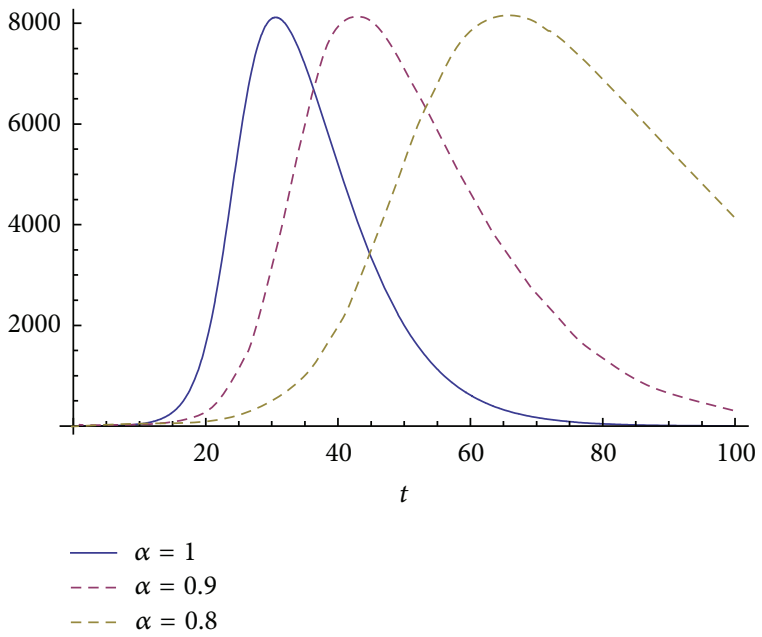

FIGURE 5: $I_{h}(t)$ for $\alpha=1,0.9,0.8, \mu_{h}=0.0000457 ; \mu_{v}=0.25 ; b=0.5$; $\beta_{h}=0.75 ; \beta_{v}=1 ; \gamma=0.1428 ; N_{h}=10000 ; B=5000$.

The approximate solutions $S_{h}(t), I_{h}(t)$, and $I_{v}(t)$ are displayed in Figures 4 and 5 with different values of $\alpha$. In each figure three different values of $\alpha$ are considered. When $\alpha=1$, system (4) is the classical integer-order system (1). In Figure 4, the variation of $S_{h}(t)$ versus time $t$ is shown for different values of $\alpha=1,0.9,0.8$ by fixing other parameters. It is revealed that $S_{h}$ does not drop significantly in a relatively small period of time for small values. Figure 5 depicts $I_{h}(t)$ versus time $t$. As mentioned by $[22,23]$, one should note that although the equilibrium points are the same for both integer order and fractional order models the solution of the fractional order model tends to the fixed point over a longer period of time. One also needs to mention that when dealing with real life problems, the order of the system can be determined by using the collected data. 


\section{Conflict of Interests}

The authors declare that there is no conflict of interests regarding the publication of this paper.

\section{Acknowledgments}

This project was funded by the Deanship of Scientific Research (DSR), King Abdulaziz University, under Grant no. 3-130/1433HiCi. The authors, therefore, acknowledge with thanks DSR technical and financial support.

\section{References}

[1] H. S. Rodrigues, M. T. Monteiro, and D. F. M. Torres, "Sensitivity analysis in a dengue epidemiological model," Conference Papers in Mathematics, vol. 2013, Article ID 721406, 7 pages, 2013.

[2] WHO, Dengue: Guidelines for Diagnosis, Treatment, Prevention and Control, World Health Organization, Geneva, Switzerland, 2nd edition, 2009.

[3] L. Esteva and C. Vargas, "Analysis of a dengue disease transmission model," Mathematical Biosciences, vol. 150, no. 2, pp. 131151, 1998.

[4] A. A. Kilbas, H. M. Srivastava, and J. J. Trujillo, Theory and Application Fractional Differential Equations, Elseviesr, Amsterdam, The Netherlands, 2006.

[5] I. Podlubny, Fractional Differential Equations, Academic Press, New York, NY, USA, 1999.

[6] S. Pooseh, H. S. Rodrigues, and D. F. M. Torres, "Fractional derivatives in dengue epidemics," in Proceedings of the International Conference on Numerical Analysis and Applied Mathematics: Numerical Analysis and Applied Mathematics (ICNAAM '11), pp. 739-742, September 2011.

[7] R. Anderson and R. May, Infectious Disease of Humans, Dynamics and Control, Oxford University Press, Oxford, UK, 1995.

[8] E. H. Elbasha, C. N. Podder, and A. B. Gumel, "Analyzing the dynamics of an SIRS vaccination model with waning natural and vaccine-induced immunity," Nonlinear Analysis: Real World Applications, vol. 12, no. 5, pp. 2692-2705, 2011.

[9] H. Hethcote, M. Zhien, and L. Shengbing, "Effects of quarantine in six endemic models for infectious diseases," Mathematical Biosciences, vol. 180, pp. 141-160, 2002.

[10] R. Gorenflo, J. Loutschko, and Y. Luchko, "Computation of the Mittag-Lefflerfunction $E_{\alpha, \beta}(z)$ and its derivatives," Fractional Calculus and Applied Analysis, vol. 5, pp. 491-518, 2002.

[11] E. Ahmed, A. M. A. El-Sayed, A. E. M. El-Mesiry, and H. A. A. El-Saka, "Numerical solution for the fractional replicator equation," International Journal of Modern Physics C, vol. 16, no. 7, pp. 1017-1025, 2005.

[12] E. Ahmed, A. M. A. El-Sayed, and H. A. A. El-Saka, "On some Routh-Hurwitz conditions for fractional order differential equations and their applications in Lorenz, Rössler, Chua and Chen systems," Physics Letters A: General, Atomic and Solid State Physics, vol. 358, no. 1, pp. 1-4, 2006.

[13] E. Ahmed, A. M. A. El-Sayed, and H. A. A. El-Saka, "Equilibrium points, stability and numerical solutions of fractionalorder predator-prey and rabies models," Journal of Mathematical Analysis and Applications, vol. 325, no. 1, pp. 542-553, 2007.

[14] D. Matignon, "Stability results for fractional differential equations with applications to control processing," in Proceedings of the Computational Engineering in Systems Applications, vol. 2, pp. 963-968, Lille, France, 1996.

[15] K. Diethelm, "An algorithm for the numerical solution of differential equations of fractional order," Electronic Transactions on Numerical Analysis, vol. 5, pp. 1-6, 1997.

[16] K. Diethelm and N. J. Ford, "Analysis of fractional differential equations," Journal of Mathematical Analysis and Applications, vol. 265, no. 2, pp. 229-248, 2002.

[17] Y. Ding and H. Ye, "A fractional-order differential equation model of HIV infection of CD4+ T-cells," Mathematical and Computer Modelling, vol. 50, no. 3-4, pp. 386-392, 2009.

[18] H. Ye and Y. Ding, "Nonlinear dynamics and chaos in a fractional-order HIV model," Mathematical Problems in Engineering, vol. 2009, Article ID 378614, 12 pages, 2009.

[19] C. Li and C. Tao, "On the fractional Adams method," Computers and Mathematics with Applications, vol. 58, no. 8, pp. 1573-1588, 2009.

[20] K. Diethelm, N. J. Ford, and A. D. Freed, "A predictor-corrector approach for the numerical solution of fractional differential equations," Nonlinear Dynamics, vol. 29, no. 1-4, pp. 3-22, 2002.

[21] E. Soewono and A. K. Supriatna, "A Two-dimensional model for transmission of dengue fever disease," Bulletin of the Malaysian Mathematical Sciences Society, vol. 24, pp. 48-57, 2001.

[22] E. Demirci, A. Unal, and N. Özalp, "A fractional order SEIR model with density dependent death rate," Hacettepe Journal of Mathematics and Statistics, vol. 40, no. 2, pp. 287-295, 2011.

[23] N. Özalp and E. Demiörciö, "A fractional order SEIR model with vertical transmission," Mathematical and Computer Modelling, vol. 54, no. 1-2, pp. 1-6, 2011. 


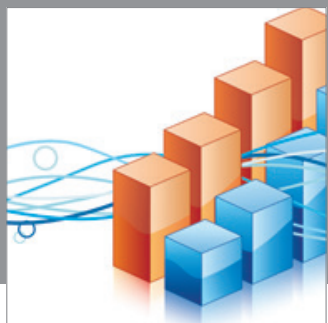

Advances in

Operations Research

mansans

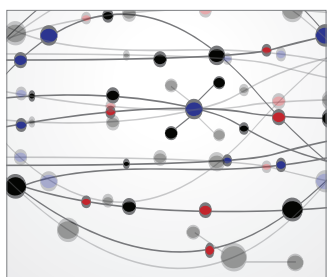

The Scientific World Journal
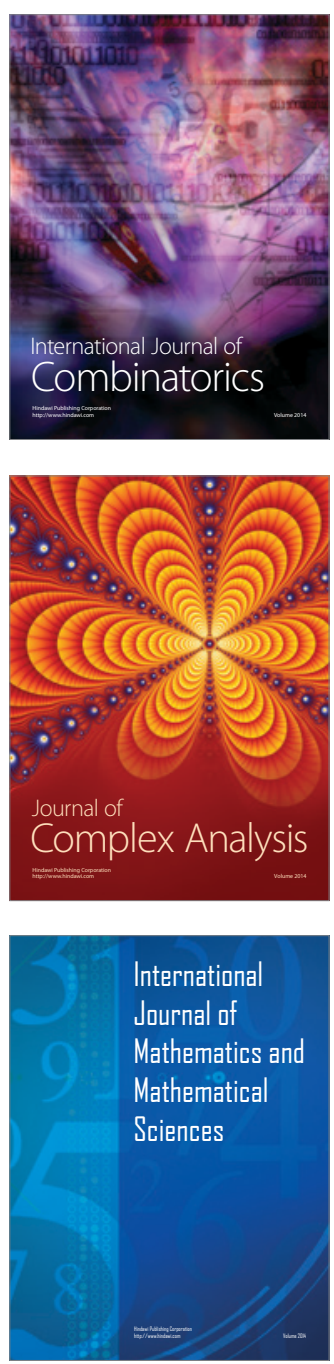
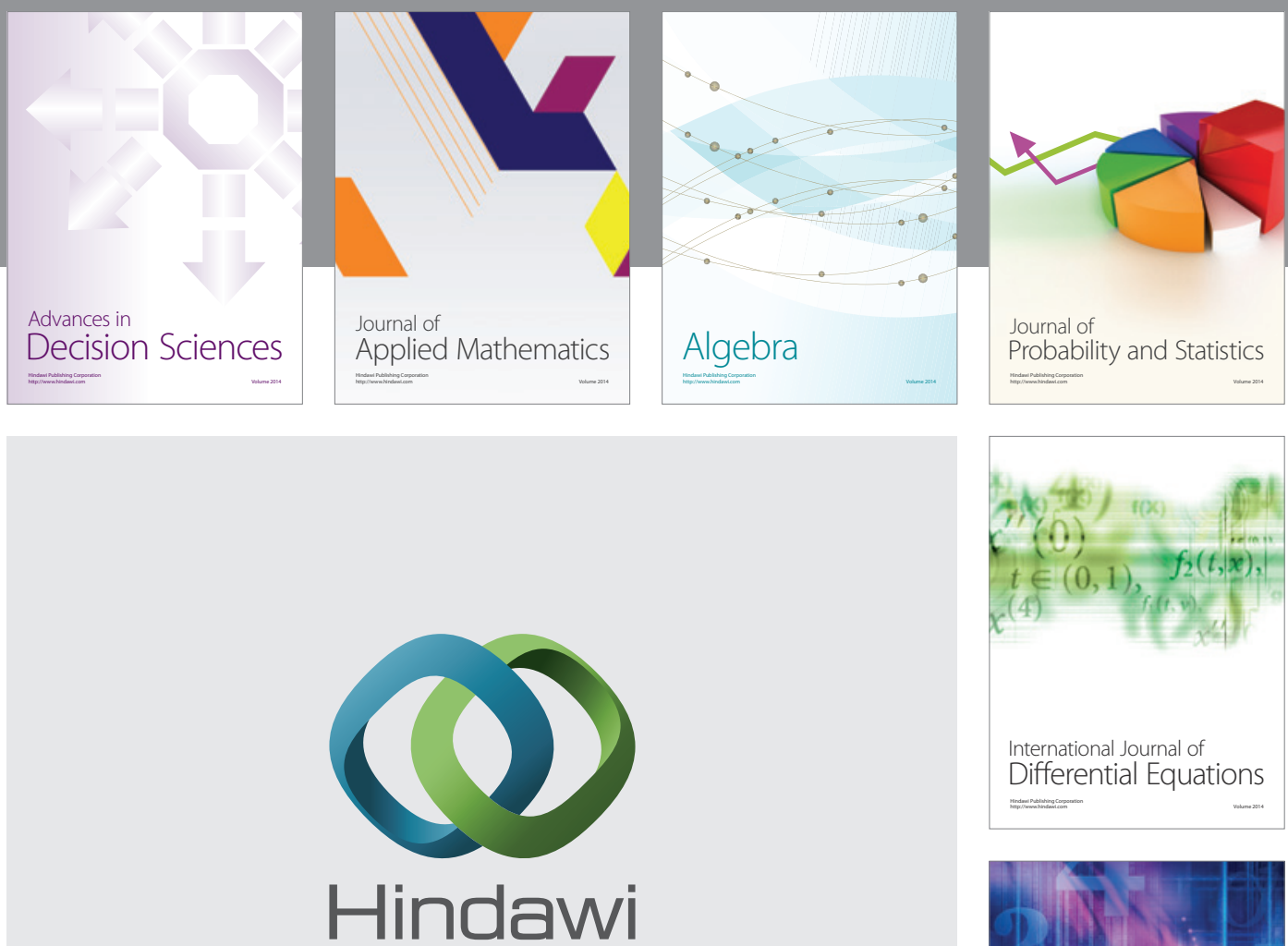

Submit your manuscripts at http://www.hindawi.com
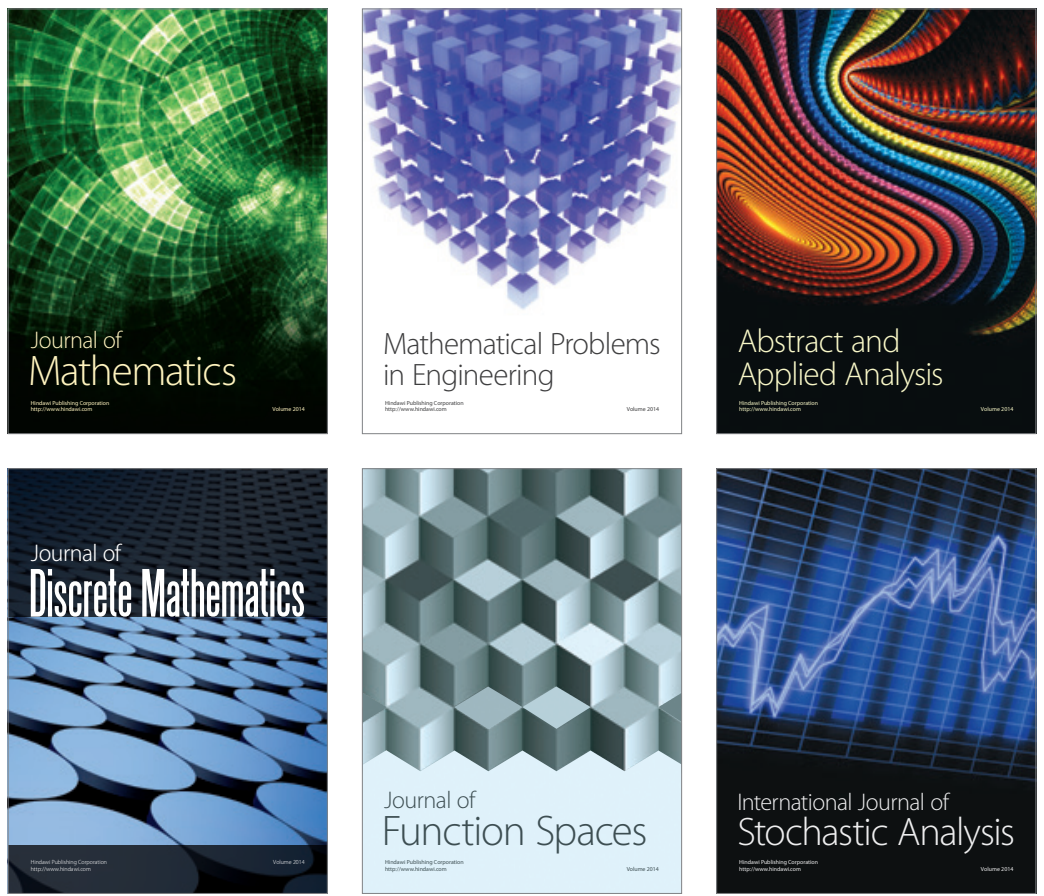

Journal of

Function Spaces

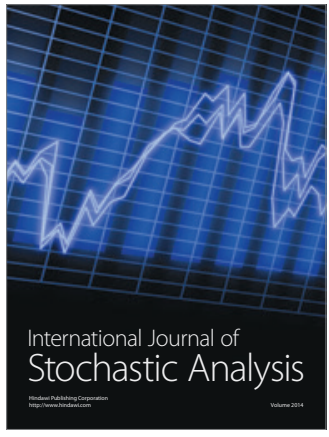

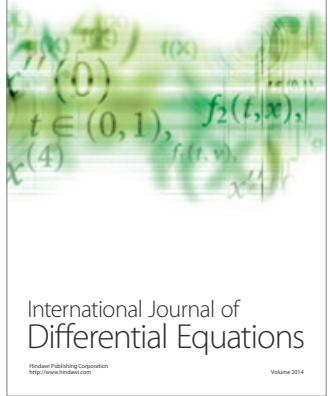
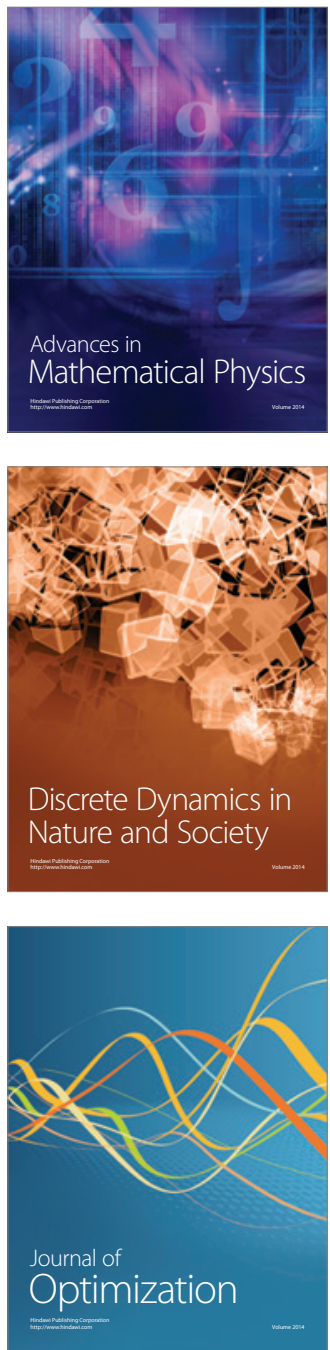